\title{
Turbulent wind waves on a water current
}

\author{
M. V. Zavolgensky ${ }^{1}$ and P. B. Rutkevich ${ }^{2}$ \\ ${ }^{1}$ Water problems Institute RAN (IVP RAN ZD), Rostov-on-Don, Russia \\ ${ }^{2}$ Space Research Institute (IKI), Moscow, Russia
}

Received: 28 July 2007 - Revised: 14 April 2008 - Accepted: 15 April 2008 - Published: 13 May 2008

\begin{abstract}
An analytical model of water waves generated by the wind over the water surface is presented. A simple modeling method of wind waves is described based on waves lengths diagram, azimuthal hodograph of waves velocities and others. Properties of the generated waves are described. The wave length and wave velocity are obtained as functions on azimuth of wave propagation and growth rate. Motionless waves dynamically trapped into the general picture of three dimensional waves are described. The gravitation force does not enter the three dimensional of turbulent wind waves. That is why these waves have turbulent and not gravitational nature. The Langmuir stripes are naturally modeled and existence of the rogue waves is theoretically proved.
\end{abstract}

\section{Introduction}

Great variety of wave types in liquids have been studied since Newton, Bernulli, Euler, Lagrange, Laplace, Cauchy, Airy, Lamb, Poincare, Lyapunov and many others. The role of the waves in the fluid dynamics theory is well understood. Remarkable advantages for the hundreds of years have been achieved in understanding of the wave properties. The main directions of research in the fluid wave motions cover (though not limited to) gravitational waves, internal waves, ship waves, tidal waves, acoustic, shock, solitary, convective waves, etc.

The investigation methods are not as diverse as the waves themselves. The basic tools of investigation are the statistics methods, approximation calculus and classical Euler equations (Lamb, 1932; Stocker, 1957). Not many specialists use the model of viscous fluid in their investigation (see, for example, Sajjadi, 1999). Sometimes Lagrange variables

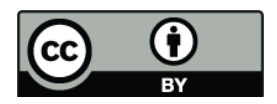

Correspondence to: P. B. Rutkevich (peter@d902.iki.rssi.ru)
(Monin, 1999), and the conformal mapping theory are applied for the waves modeling (Nekrasov, 1961).

However, despite such an intense intellectual attack there still exist issues not well understood in the wave motions theory. One of them is the theory of wind waves on the water surface which is usually investigated based on the ideal fluid theory and neglecting the viscosity. In other cases numerical, experimental, semiempirical, probabilistic, statistic methods are used in investigations. All researches assume that the wind waves are gravitational. Therefore, nowadays there does not exist any analytical model of the wind waves with account of the fluid viscosity.

The idea of modeling of the wind waves neglecting the influence of viscosity between the water surface and the air wind seems hopeless since the wind is the generator of these waves. However, there is a strong reason to neglect the viscosity, and it is due to the internal structure of the NavierStokes equations. Indeed, in order to take into account analytically the wind influence on the water surface one has to assume that the location of the main masses of air driven by the wind is high enough as compared to the surface wavelengths. As a matter of fact this condition is always satisfied in nature. To model the wind waves in viscous fluid one should also assume that the waves do not affect the water current in the deep stream, existing in the sea. In other words undisturbed deep water flow in the first approximation should be located infinitely far from the water surface. And again, in the first approximation the fluid should be considered infinitely deep. And the reason why the wind waves cannot be described on the base of the Navier-Stokes equations is that the Navier-Stokes equations do not have a stationary solution for the fluid flow in the half-space under influence of stationary tangent stresses on its surface (Kochin et al., 1963; Kondrat'ev et al., 1990).

The idea that conventional Navier-Stokes equations are not complete has been mentioned before. For example, (Birkhoff, 1960) mentions that the NavierStokes equations

Published by Copernicus Publications on behalf of the European Geosciences Union. 


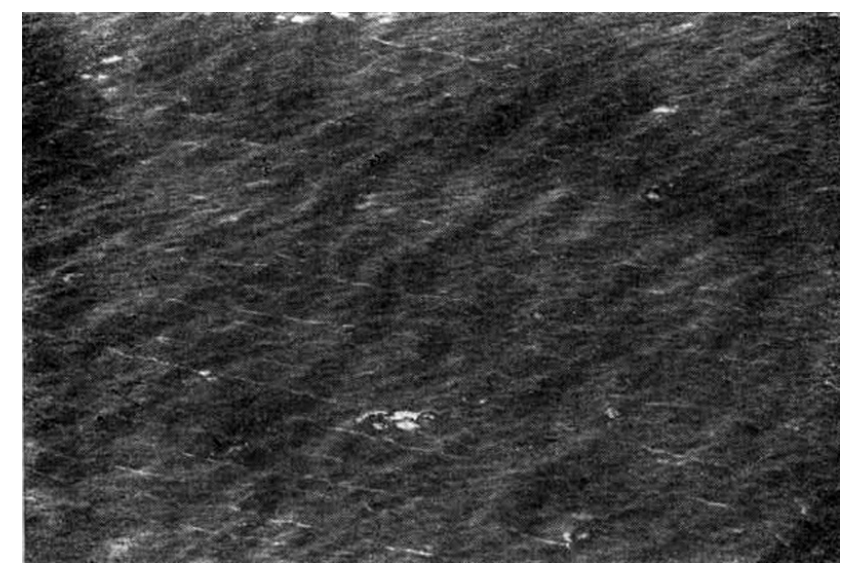

Fig. 1. Air photograph of the sea surface at strong wind. The light Langmuir stripes and the dark stripes of wind wave troughs can be observed (Titov, 1969).

describe well enough incompressible flows of viscous fluids, to which belong usual gases and liquids at speeds considerably smaller speed of sound. However description of turbulent flows requires accurate use of statistical analysis at high mathematical level. Therefore, instead of use of the NavierStokes equations at formulation of obvious boundary problems (for example, the problems of stationary current) it is necessary to address directly to the physical validity for statement of the appropriate boundary problem. In our model we use the turbulent resistance to resolve this problem.

General structure of the surface waves generated by the wind can be understood even from a photo image of the sea surface Fig. 1 (Titov, 1969). One can notice distinct directions of bright Langmuir stripes and the direction at which the waves do not propagate. Motionless waves correspond to the zero wavelength in the corresponding direction. Therefore, there is a closed curved line the tangent to which is aligned in the direction of no wave propagation (since the waves are confined such a curve does not go to infinity). The chords lengths of such a line counted from the point of contact are equal to the length of wind waves. It is appropriate to call this curve as wavelength diagram along the azimuth of the chosen diagram chord. It is appropriate to call this curve azimuth wind waves velocity hodograph along the azimuth of the chosen hodograph chord.

The Langmuir stripes represent motionless waves since they are in fact motionless on the water surface. These waves "propagate" (i.e. are oriented) in the direction orthogonal to the Langmuir stripes. It is clear then that along the orientation of the motionless waves (orthogonal to the Langmuir stripes), the velocity of the wind eaves equals zero.

The velocity hodograph and the wavelength diagram of the wind waves really exist and will described later in this paper. The model of the wind waves is based on the Navier-Stokes equation with isotropic turbulent resistance. The presented analytical model of turbulent wind waves over the water current is remarkably simple, since the waves are described through elementary relations. Moreover, the model predicts existence of the new waves: motionless and barchan's waves. The model also describes the choppy sea the kind of waves that up to now have no analytical description.

\section{Basic dispersion relations}

As it has already been mentioned above, Navier-Stokes equations do not have a stable solution in a semi-space with tangent stress on the surface. However, if the deep water current and high air flow have different directions there should exist a stable solution, responsible for wind-generated waves on the surface.

We start from the Navier-Stokes equations with turbulent resistance (Gill, 1982; Lamb, 1932; Kondrat'ev et al., 1990; Struminskii, 1969):

$$
\frac{\partial \boldsymbol{v}}{\partial t}+(\boldsymbol{V} \cdot \nabla) \boldsymbol{v}=-\frac{\nabla p}{\rho}+\nu \nabla^{2} \boldsymbol{v}-\kappa(\boldsymbol{v}-\boldsymbol{V})+\boldsymbol{g}, \nabla \cdot \boldsymbol{v}=0,
$$

where $\boldsymbol{V}$ is the fluid velocity at infinity, and $\kappa$ is turbulent resistance of the medium. Equation (1) has to be applied twice, first for the air in the upper half-space, and second for the water in the bottom half-space, and satisfy the boundary conditions between these two regions.

Introducing Cartesian coordinates $O x^{\prime} y^{\prime} z^{\prime}$, with axis $O z^{\prime}$ directed against the gravity force $\boldsymbol{g}$, and axis $O x^{\prime}$ directed along the direction of the constant air velocity vector $\boldsymbol{V}_{1}$ at $z^{\prime} \rightarrow \infty$. Density, kinematic viscosity and turbulent resistance of the air denote as $\rho_{1}, v_{1}$ and $\kappa_{1}$, respectively, finally velocity and hydrodynamic pressure of the air as $\boldsymbol{v}_{1}^{\prime}\left(x^{\prime}, y^{\prime}, z^{\prime}, t^{\prime}\right)$ and $p_{1}^{\prime}\left(x^{\prime}, y^{\prime}, z^{\prime}, t^{\prime}\right)$. Similarly, water is described by the parameters $\rho, v$ and $\kappa,\left(\rho \gg \rho_{1}\right)$, velocity $\boldsymbol{v}^{\prime}\left(x^{\prime}, y^{\prime}, z^{\prime}, t^{\prime}\right)$, pressure $p^{\prime}\left(x^{\prime}, y^{\prime}, z^{\prime}, t^{\prime}\right)$ and at $z^{\prime} \rightarrow-\infty$ moves with constant velocity $\boldsymbol{V}_{0}=V_{0}(\boldsymbol{i} \cos \alpha+\boldsymbol{j} \sin \alpha)$, where $\boldsymbol{i}$ and $\boldsymbol{j}$ are the unit vectors along $O x^{\prime}$ and $O y^{\prime}$, respectively. The angle $\alpha$ is counted from vector $\boldsymbol{V}_{1}$ towards $\boldsymbol{V}_{0}(-\pi \leq \alpha \leq \pi)$. The water-air interface plane is $z^{\prime}=\zeta^{\prime}\left(x^{\prime}, y^{\prime}, t^{\prime}\right)$. Therefore, to determine the parameters $\boldsymbol{v}^{\prime}{ }_{1}, p_{1}^{\prime}, \boldsymbol{v}^{\prime}, p^{\prime}$ and $\zeta^{\prime}$ we have Eq. (1) with isotropic turbulent resistance for air:

$$
\begin{aligned}
& \frac{\partial \boldsymbol{v}^{\prime}{ }_{1}}{\partial t^{\prime}}+\left(\boldsymbol{v}_{1}^{\prime} \cdot \nabla\right) \boldsymbol{v}_{1}^{\prime}=-\frac{\nabla p_{1}^{\prime}}{\rho_{1}}+v_{1} \nabla^{2} \boldsymbol{v}_{1}^{\prime}{ }_{1} \\
& -\kappa_{1}\left(\boldsymbol{v}_{1}^{\prime}-\boldsymbol{V}_{1}\right)-\boldsymbol{g}, \quad \nabla \cdot \boldsymbol{v}_{1}^{\prime}=0 \quad\left(z^{\prime}>\zeta^{\prime}\right),
\end{aligned}
$$

and water:

$$
\begin{aligned}
& \frac{\partial \boldsymbol{v}^{\prime}}{\partial t^{\prime}}+\left(\boldsymbol{v}^{\prime} \cdot \nabla\right) \boldsymbol{v}^{\prime}=-\frac{1}{\rho} \nabla p^{\prime}+v \nabla^{2} \boldsymbol{v}^{\prime}-\kappa\left(\boldsymbol{v}^{\prime}-\boldsymbol{V}_{0}\right)-\boldsymbol{g}, \\
& \nabla \cdot \boldsymbol{v}^{\prime}=0 \quad\left(z^{\prime}<\zeta^{\prime}\right),
\end{aligned}
$$

supported by the fluxes continuity conditions at the air-water boundary $z^{\prime}=\zeta^{\prime}$ (i.e. equality of their velocities and stresses 
along the tangent direction), kinematic condition, that the interface points do not leave this interface and conditions at infinity. This system of equations with the mentioned boundary conditions has a stationary solution at the boundary,

$\boldsymbol{v}_{1}^{\prime}=V_{*} \boldsymbol{v}_{10}, \quad \boldsymbol{v}^{\prime}=V_{*} \boldsymbol{v}_{0}, \quad p_{1}^{\prime}=p_{0}-\rho_{1} g z^{\prime}$,

$p^{\prime}=p_{0}-\rho g z^{\prime}, \quad \zeta^{\prime}=0, v_{10}=i \Phi+j \Psi, \boldsymbol{v}_{0}=\boldsymbol{i} \varphi+\boldsymbol{j} \psi$,

$p_{0}=$ const, $z=z^{\prime} \sqrt{\frac{\kappa}{v}}, \Lambda=\sqrt{\frac{\kappa_{1} v}{\kappa \nu_{1}}}$,

$\varphi=\frac{V_{0} \cos \alpha}{V_{*}}-\frac{\delta\left(V_{0} \cos \alpha-V_{1}\right)}{V_{*}(1+\delta)} \mathrm{e}^{z}, \quad(z<0)$,

$\psi=\frac{V_{0} \sin \alpha}{V_{*}}-\frac{\delta V_{0} \sin \alpha}{V_{*}(1+\delta)} \mathrm{e}^{z}, \quad(z<0)$,

$\Phi=\frac{V_{1}}{V_{*}}+\frac{V_{0} \cos \alpha-V_{1}}{V_{*}(1+\delta)} \mathrm{e}^{-\Lambda z}, \quad(z>0)$,

$\Psi=\frac{V_{0} \sin \alpha}{V_{*}(1+\delta)} \mathrm{e}^{-\Lambda z}, \quad(z>0), \quad \delta=\frac{\rho_{1} \sqrt{\nu_{1} \kappa_{1}}}{\rho \sqrt{\nu \kappa}}$.

At large wind $V_{1}$ or water $V_{0}$ velocities the solution (2) loses its stability. Therefore we are looking for the solution of the problem different from (2):

$\boldsymbol{v}_{1}^{\prime}=V_{*}\left(\boldsymbol{v}_{10}+\boldsymbol{V}\right), \quad \boldsymbol{v}^{\prime}=V_{*}\left(\boldsymbol{v}_{0}+\boldsymbol{v}\right), \quad \boldsymbol{V}=\{\tilde{U}, \tilde{V}, \tilde{W}\}$,

$\boldsymbol{v}=\{\tilde{u}, \tilde{v}, \tilde{w}\} p_{1}^{\prime}=p_{0}-\rho_{1} g z^{\prime}+P_{1 *} \tilde{P}$,

$p^{\prime}=p_{0}-\rho g z^{\prime}+P_{*} \tilde{p}, \quad \zeta^{\prime}=Z_{*} \tilde{\zeta}(x, y, t)$,

$\{x, y, z\}=\left\{x^{\prime}, y^{\prime}, z^{\prime}\right\} \sqrt{\kappa / \nu}, \quad t=t^{\prime} \kappa$

$P_{1 *}=\rho_{1} v_{1} \kappa, \quad P_{*}=\rho \nu \kappa, \quad Z_{*}=\sqrt{\nu / \kappa}$.

All the functions marked with tilde $(\sim)$ (except $\tilde{\zeta})$ depend on $x, y, z$ and $t$. New variables ( 3 will convert initial equations into a dimensionless form, After linearization, the initial equations and limiting conditions (2) for new variables $\boldsymbol{V}, \boldsymbol{v}, \tilde{P}, \tilde{p}$ and $\tilde{\zeta}$ satisfy the following equations:

$$
\begin{aligned}
& R\left[\frac{\partial \boldsymbol{V}}{\partial t}+\Phi \frac{\partial \boldsymbol{V}}{\partial x}+\Psi \frac{\partial \boldsymbol{V}}{\partial y}+\left(\boldsymbol{i} \frac{\mathrm{d} \Phi}{\mathrm{d} z}+\boldsymbol{j} \frac{\mathrm{d} \Psi}{\mathrm{d} z}\right) \tilde{W}\right]= \\
& =-\nabla \tilde{\boldsymbol{P}}+\nabla^{2} \boldsymbol{V}-\Lambda^{2} \boldsymbol{V}, \quad \nabla \boldsymbol{V}=0(z>0) \\
& \frac{\partial \boldsymbol{v}}{\partial t}+\varphi \frac{\partial \boldsymbol{v}}{\partial x}+\psi \frac{\partial \boldsymbol{v}}{\partial y}+\left(\boldsymbol{i} \frac{\mathrm{d} \varphi}{\mathrm{d} z}+\boldsymbol{j} \frac{\mathrm{d} \psi}{\mathrm{d} z}\right) \tilde{w}= \\
& =-\nabla \tilde{p}+\nabla^{2} \boldsymbol{v}-\boldsymbol{v}, \quad \nabla \boldsymbol{v}=0(z<0), \quad R=\frac{v}{v_{1}} \\
& z=0: \frac{\partial \tilde{\zeta}}{\partial t}+\varphi \frac{\partial \tilde{\zeta}}{\partial x}+\psi \frac{\partial \tilde{\zeta}}{\partial y}=\tilde{w}=\tilde{W}
\end{aligned}
$$

$\tilde{u}=\tilde{U}, \tilde{v}=\tilde{V}, \boldsymbol{V}=0(z=+\infty), \boldsymbol{v}=0(z=-\infty)$

$$
\begin{aligned}
& z=0: \sigma \tilde{\zeta}-\tilde{p}+2 \frac{\partial \tilde{w}}{\partial z}=\varepsilon\left(2 \frac{\partial \tilde{W}}{\partial z}-\tilde{P}\right) \\
& \sigma=\frac{g}{\kappa \sqrt{\kappa \nu}}\left(1-\frac{\rho_{1}}{\rho}\right), \quad \varepsilon=\frac{\rho_{1} \nu_{1}}{\rho \nu} \ll 1 \\
& z=0: \frac{\partial \tilde{u}}{\partial z}+\frac{\partial \tilde{w}}{\partial x}=\varepsilon\left(\frac{\partial \tilde{U}}{\partial z}+\frac{\partial \tilde{W}}{\partial x}\right), \\
& \frac{\partial \tilde{v}}{\partial z}+\frac{\partial \tilde{w}}{\partial y}=\varepsilon\left(\frac{\partial \tilde{V}}{\partial z}+\frac{\partial \tilde{W}}{\partial y}\right)
\end{aligned}
$$

where $V_{*}=\sqrt{\kappa v}$.

Omitting the terms of the order of $O(\varepsilon)$, and having calculated $\tilde{u}, \tilde{v}$ and $\tilde{w}$ in order to determine $\tilde{P}, \tilde{U}, \tilde{V}, \tilde{W}$ we obtain a nonhomogeneous boundary problem. We are not going to investigate this problem since the water waves are more interesting then those in the air over the water surface. We are looking for the solution of the matrix $\{\tilde{u}, \tilde{v}, \tilde{w}, \tilde{p}, \tilde{\zeta}\}$ in the form

$\{\tilde{u}, \tilde{v}, \tilde{w}, \tilde{p}, \tilde{\zeta}\}=\{u(z), v(z), w(z), p(z), \zeta\} \mathrm{e}^{[\mathrm{i}(m x+n y-c t)]}$,

$$
\zeta=\mathrm{const}
$$

where $\mathrm{i}=\sqrt{-1}$.

After substitution into (4) one obtains the following differential equations for unknown variables $u, v, w$ and $p$ (for simplicity we used a vector representation for three variables and three equations):

$$
\begin{array}{r}
\mathrm{i}(m \varphi+n \psi)\{u, v, w\}+w\left\{\varphi^{\prime}, \psi^{\prime}, 0\right\}= \\
=-\left\{\mathrm{i} m p, \mathrm{i} n p, p^{\prime}\right\}+L\{u, v, w\},
\end{array}
$$

$w^{\prime}+\mathrm{i} m u+\mathrm{i} n v=0, \quad(z=0), \quad L=\frac{\mathrm{d}^{2}}{\mathrm{~d} z^{2}}-k^{2}-1+\mathrm{i} c$,

$$
k=\sqrt{m^{2}+n^{2}} .
$$

Here the prime stands for derivative with respect to $z$. Multiplying the first component of the vector row (6) by im, the second by in and add together the first two components of the obtained relation. Then taking into account the continuity equation we obtain

$k^{2} p=L w-(m \varphi+n \psi) w^{\prime}+\left(m \varphi^{\prime \prime}+n \psi^{\prime \prime}\right) w=0,(z<0)(7)$

Now calculate the derivative $p^{\prime}$ and put it equal to the same derivative from the third row of the vector Eq. (6). This gives the equation for determination the component $w(z<0)$ :

$[L-\mathrm{i}(m \varphi+n \psi)]\left(w^{\prime \prime}-k^{2} w\right)+\mathrm{i}\left(m \varphi^{\prime \prime}+n \psi^{\prime \prime}\right) w=0$

Multiplying the first component of the vector equation (6) by $n$, the second by $m$ and subtracting the first from the second yields $(z<0)$

$[L-\mathrm{i}(m \varphi+n \psi)](n u-m v)=\left(m \varphi^{\prime}-m \psi^{\prime}\right) w$, 
$m u+n v=\mathrm{i} w^{\prime}$

The limiting conditions for the Eqs. (8), (9) are obtained by substitution of the solution (5) into the limiting conditions (4) at $\varepsilon=0$ :

$\mathrm{i} \zeta(m \varphi+n \psi-c)=w, \quad \sigma \zeta=p-2 w^{\prime}$,

$u^{\prime}+\mathrm{i} m w=v^{\prime}+\mathrm{inw} \quad(z=0)$

So $(n u-m v)^{\prime}=0 \quad(z=0)$ and $n u-m v=0 \quad(z=-\infty)$. Excluding the parameter $\zeta$ from the two remained limiting conditions and using the continuity Eq. (6) and the Eq. (7), one obtains the limiting conditions for the Eq. (7), that is formulate the boundary problem for determining the amplitude of the vertical velocity $w(z)$ :

$\left(\mathrm{i} L+m \varphi+n \psi-2 \mathrm{i} k^{2}\right) w^{\prime}=\left(m \varphi^{\prime}+n \psi^{\prime}+k^{2} \sigma\right) w, \quad(z=0)$

$w^{\prime \prime}+k^{2} w=0(z=0), \quad w=0 \quad(z=-\infty)$.

The amplitudes of $u, v, p$ and $\zeta$ are not necessary for obtaining the dispersion relation. These amplitudes can be derived later from the nonhomogeneous problem for the Eq. (9) after the component $w$ is found. The dispersion relation connects real wavenumbers $m$ and $n$ with the complex frequency $c$, it is derived from the spectral problem (8),(10), that can be solved approximately using the following idea. The equation coefficients (8) on account of Eq. (2) are linear functions of $\exp (z),(z<0)$. The parameter $z=z^{\prime} \sqrt{\kappa / v}$ for finite $z^{\prime}$ is large, since the turbulent resistance is strong during the wave formation. So the exponents in the Eq. (8) coefficients are the pronounced interface layers. For derivation of the dispersion relation these layers are taken into account by replacement the exponents with units. In the boundary conditions (10) which define the dispersion relation, the influence of the layers is taken into account more precisely. The so approximated spectral problem (8), (10) takes the form

$$
\begin{aligned}
& \left(\frac{\mathrm{d}^{2}}{\mathrm{~d} z^{2}}-q\right)\left(\frac{\mathrm{d}^{2}}{\mathrm{~d} z^{2}}-k^{2}\right) w+\mathrm{i} \delta \mathcal{B} w=0,(z<0), \\
& q=k^{2}+1-\mathrm{i} c-\mathrm{i} \delta \mathcal{B}, \\
& {\left[\left(L-2 k^{2}-\mathrm{i} b\right) w^{\prime}+\mathrm{i} \delta \mathcal{B} w\right](b-c)+\mathrm{i} k^{2} \sigma w=0}
\end{aligned}
$$

$w^{\prime \prime}+k^{2} w=0 \quad(z=0),\left.\quad w\right|_{z=-\infty}=0$

$b=\frac{\delta m V_{1}+(m \cos \alpha+n \sin \alpha) V_{0}}{(1+\delta) \sqrt{\kappa \nu}}$,

$\mathcal{B}=\frac{m V_{1}-(m \cos \alpha+n \sin \alpha) V_{0}}{(1+\delta) \sqrt{\kappa v}}$.

Solution of the Eq. (11) vanishing at $z \rightarrow-\infty$ has the form $w=C_{1} \exp \left(\xi_{1} z\right)+C_{2} \exp \left(\xi_{2} z\right), \quad(z<0)$, where $\xi_{1}, \xi_{2}$ are the roots of characteristic equation:

$\xi^{4}-\left(q+k^{2}\right) \xi^{2}+q k^{2}+\mathrm{i} \delta \mathcal{B}=0$

with negative sign of real parts. Substituting velocity (12) into the boundary conditions (11) at $z=0$, one gets a system of two linear homogeneous algebraic equations for variables $C_{1}$ and $C_{2}$. The determinant of this system is must be equal to zero, thus giving following dispersion relation is obtained

$$
\begin{aligned}
& \left(\xi_{1}-\xi_{2}\right)\left\{\left[(b-c)\left(q+3 k^{2}\right) \xi_{1} \xi_{2}-\mathrm{i} \delta \mathcal{B}\left(\xi_{1}+\xi_{2}-1\right)+\right.\right. \\
& \left.\left.+k^{2}\left(q-k^{2}\right)\right]-\mathrm{i} k^{2} \sigma\left(\xi_{1}+\xi_{2}\right)\right\}=0 \\
& \xi_{1} \xi_{2}=\sqrt{q k^{2}+\mathrm{i} \delta \mathcal{B}}, \quad \xi_{1}+\xi_{2}=\sqrt{q+k^{2}+2 \xi_{1} \xi_{2}},
\end{aligned}
$$

$\operatorname{Re} \sqrt{z} \geq 0$

The most important is the situation when $\xi_{1}=\xi_{2}$ since in the case of multiple roots of the Eq. (13) the disturbances (12) penetrate into water deeper than the disturbances of two different root. At $\xi_{1}=\xi_{2}$ instead the relation (12) one has to use $w=\left(C_{1}+C_{2} z\right) \exp \left(\xi_{1} z\right),(z<$ $0)$, which maximal value modulating the oscillations is attained bellow the surface, rather than on the surface as in the case of relations (12). The roots of Eq. (13) are equal at $\left(q+k^{2}\right)^{2}=4\left(q k^{2}+\mathrm{i} \delta \mathcal{B}\right)$. Therefore the parameter $\left(q=k^{2} \pm \sqrt{\mathrm{i} \delta \mathcal{B}},(\operatorname{Re} \sqrt{z} \geq 0)\right)$. Taking into account the parameter $q$ definition (11) one obtains the complex oscillation frequency (5): $\left(c=c_{r}+\mathrm{i} c_{i}=b-\mathrm{i} \pm(\mathrm{i}-\operatorname{sgn}(\mathcal{B})) \sqrt{\in \delta|\mathcal{B}|}\right)$ $(\operatorname{sgn} x=1$ at $x>0, \operatorname{sgn}(x)=-1$ for $x<0)$. Hence the frequency $c_{r}$ and the growth rate $c_{i}$ of the waves (5), corresponding to the dispersion relation at $\xi_{1}=\xi_{2}$ become

$c_{r}=b \mp \sqrt{2 \delta \mathcal{B}} \operatorname{sgn} \mathcal{B}, \quad c_{i}=-1 \pm \sqrt{2 \delta \mathcal{B}}$.

Here one has to use simultaneously either the upper or the lower signs.

One can see, that there is no gravity force $g$ in the dispersion relations (15), thus the waves described by relation (15) can be called as non-gravitational. Analysis presented in the rest of this work is based on the relation (15).

\section{Length and velocity of the wind waves}

Introducing the azimuth $\theta$ of the wave vector $\boldsymbol{k}=\{m, n\}$ with respect to the wind velocity $\boldsymbol{V}_{1}$, one obtains $m=k \cos \theta$, $n=k \sin \theta$, and the relations (11) for $b$ and $\mathcal{B}$ can be rewriten as

$$
\begin{aligned}
& b=k \frac{\sqrt{A} \sin (\theta+\gamma)}{(1+\delta) \sqrt{\kappa \nu}}, \quad 2 \delta \mathcal{B}=k \frac{\sqrt{C} \sin (\beta-\theta)}{(1+\delta) \sqrt{\kappa \nu}}, \\
& A=\delta^{2} V_{1}^{2}+2 \delta V_{1} V_{0} \cos \alpha+V_{0}^{2}, \\
& C=4 \delta^{2}\left(V_{1}^{2}-2 V_{1} V_{2} \cos \alpha+V_{0}^{2}\right),
\end{aligned}
$$


$\sin \beta=\frac{2 \delta\left(V_{1}-V_{0} \cos \alpha\right)}{\sqrt{C}}, \cos \beta=\frac{2 \delta V_{0} \sin \alpha}{\sqrt{C}}$,

$\sin \gamma=\frac{\delta V_{1}+V_{0} \cos \alpha}{\sqrt{A}}, \cos \gamma=\frac{V_{0} \sin \alpha}{\sqrt{A}}$.

Now from (15)

$\frac{c_{r}}{k}=\frac{\sqrt{A} \sin (\theta+\gamma)}{(1+\delta) \sqrt{\kappa v}}+\frac{c_{i}+1}{k} \operatorname{sgn} \sin (\beta-\theta)$,

$k=\frac{(1+\delta)\left(c_{i}+1\right)^{2} \sqrt{\kappa \nu}}{\sqrt{C}|\sin (\beta-\theta)|}$.

The waves defined by the expressions (17) can be called as $\kappa$-waves, since they have turbulent and not gravitational nature (i.e. the gravity force $g$ does not appear in the formulas (17)). To describe the $\kappa$-waves let us abandon the tradition and investigate their properties not as function of the wave number $k$, but as a function of the growth rate, or, more precisely, as a function of parameter $K=c_{i}+1$. The $K$ dependence of $k$ is presented in (17). The values $K<1$ correspond to the decaying waves, the value $K=1$ corresponds to the neutral waves, and at $K>1$ there exist non decaying waves. In order to obtain the dimensional velocity $\dot{\lambda}_{K}$ from Eq. (17) and the wavelength $\lambda_{K}$ of $\kappa$-waves the ratio $c_{r} / k$ sould be multiplied by the velocity scale $\sqrt{\kappa \nu}$, and the dimensional wavelength $2 \pi / k$ by the length scale $\sqrt{\nu / \kappa}$ :

$\boldsymbol{v}_{K}=\frac{\sqrt{A} \sin (\theta+\gamma)}{1+\delta}+\frac{\sqrt{C} \sin (\beta-\theta)}{(1+\delta) K}$,

$\lambda_{K}=L_{K}|\sin (\beta-\theta)|, \quad L_{K}=\frac{2 \pi \sqrt{C}}{\kappa(1+\delta) K^{2}}$.

The formulas (18) and (19) describe the wave front velocity and the wave length at any direction $\theta$ with respect to the wave velocity. The formula (18) is convenient for description of the waves velocities at large values of the absolute value of dimensionless characteristics $K$ of the growth rate $c_{i}$. It follows, for example, from formulas (18) and (19) that at large values of $|K|$ the decaying waves and the non decaying waves move at the same velocity symmetrically with respect to direction $\theta=(\pi / 2)-\gamma$, in which the velocity of the waves is maximal. Note that the wavelengths (19) of these waves are not symmetric with respect to this direction. If the parameter $|K|$ does not increase then instead of (18) for the velocities description $\kappa$-waves it is more convenient to use another expression. Let us use in (18) the definition of the parameters $A, C$ and the angles $\beta, \gamma$ from (16). Then

$$
\begin{aligned}
\boldsymbol{v}_{K} & =\frac{\delta(K-2) V_{1} \cos \theta+(K+2 \delta) V_{0} \cos (\theta-\alpha)}{(1+\delta) K}, \\
\boldsymbol{v}_{K} & =\Sigma_{K} \sin \left(\theta+\theta_{K}\right)=\left|\Sigma_{K}\right| \cos \left(\theta-\theta_{K}^{\prime}\right), \\
\Sigma_{K} & =\frac{\sqrt{A K^{2}+2 B K+C}}{(1+\delta) K}
\end{aligned}
$$

$$
\begin{aligned}
& B=2 \delta\left[V_{0}^{2}-(1-\delta) V_{1} V_{0} \cos \alpha-\delta V_{1}^{2}\right], \\
& \theta_{K}^{\prime}=\frac{\pi}{2} \operatorname{sgn}(K)-\theta_{K}, \\
& \sin \theta_{K}=\frac{K\left(\delta V_{1}+V_{0} \cos \alpha\right)-2 \delta\left(V_{1}-V_{0} \cos \alpha\right)}{\sqrt{A K^{2}+2 B K+C}}, \\
& \cos \theta_{K}=\frac{V_{0}(K+2 \delta) \sin \alpha}{\sqrt{A K^{2}+2 B K+C}}, \\
& B^{2}-4 A C=-4 \delta^{2}(1+\delta)^{2} V_{1}^{2} V_{0}^{2} \sin ^{2} \alpha \leq 0 .
\end{aligned}
$$

Here $\left|\Sigma_{K}\right|$ is the maximum velocity of the $\kappa$-waves with respect to the azimuth $\theta$ at the fixed value $K$, corresponding to waves propagation in the direction $\theta=\theta_{K}^{\prime}$. At $K=0$ the growth rate $c_{i}=-1$. It follows from (15) and (16)that in this case ether $k=0$ (there is no wave motion), or $\theta=\beta$ or $\theta=\beta+\pi$. The uncertainty zero divided by zero at $K=0$, $\theta=\beta, \theta=\beta+\pi$ in the formula (19) is prescribed the value zero, since any $\kappa$-waves at $K \quad /=0$ at the directions $\theta=\beta$, $\theta=\beta+\pi$ have zero lengths. The direction in which the developed water waves have zero lengths (that is not seen) are well known from the air photography observations (Titov, 1969). This direction corresponds to the orientation of the wind waves troughs. Formulas (16) define the $\beta$ dependence of this orientation on the values and directions of the wind and water flow velocities. Note that the directions $\theta=\beta \pm \pi / 2$ of the waves propagation of the maximal azimuth wavelength and the directions $\theta=\beta, \theta=\beta+\pi$ in which the wavelength equals zero do not depend on the growth rate. These directions do not depend even on air and water physical properties. At $K=0$ the velocity (21) and the length (19) of the $\kappa$-waves go to infinity. There takes place an internal resonance of the growth rate of the $\kappa$-waves with the turbulent resistance frequency (in this case $c_{i}=-\kappa$ ). If one expresses the parameter $K^{2}$ with the use of $L_{K}$ and the formula (19), then the approach of the parameter $K$ to zero means just increase of the maximal wind waves length.

\section{Wave lengths diagram}

The formula $\lambda_{K}=L_{K}|\sin (\theta-\beta)|$ (see Eq. 19) describing the wind wave lengths for all values of the angle $\theta$, means that the wave lengths diagram is a pair of equal circles. Let us mark an arbitrary pole $O$ on the plane $z=0$ and fix a counting out direction $\boldsymbol{V}_{1}$ of the azimuth $\theta$. For simplicity the rays on the $z$-plane are marked by their azimuths. The circles of the wind wave lengths diagram touch the straight line $\theta=\beta$, $\theta=\beta+\pi$ (Fig. 2).

The centers of the wave lengths diagram circles $C$ and $C^{\prime}$ are located on the straight line $\theta=\beta \pm \frac{1}{2} \pi$ at distance $\frac{1}{2} L_{K}$ from the diagram circles point of contact.

It follows from the formula (16) that the angle $\beta$ does not depend of the wind waves growth rate and the $\tan \beta$ does not depend of the parameter d (see Eq. 2) that is, does not 


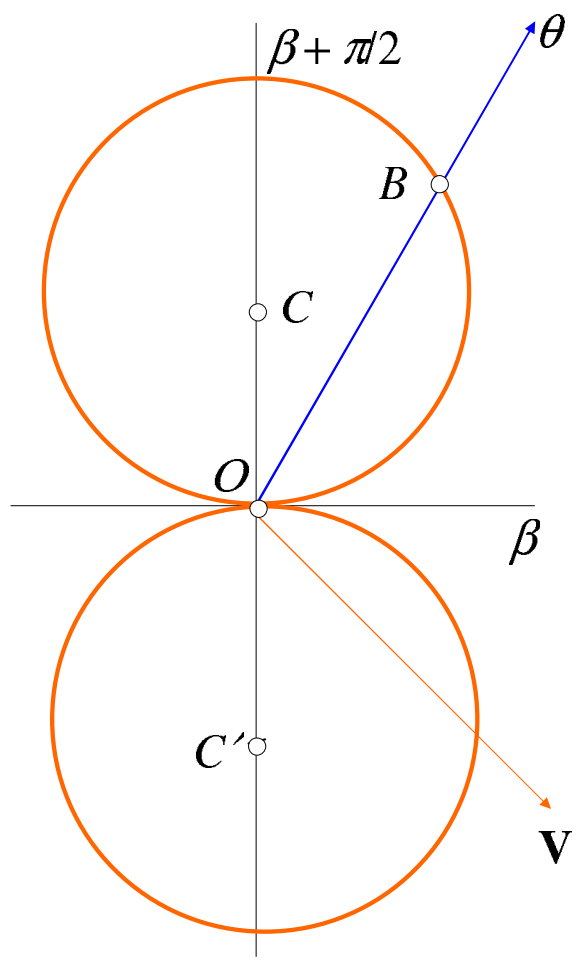

Fig. 2. Wave lengths diagram.

depend neither of the viscosity, nor of their turbulent resistances. That is why the $\beta$ angle is defined only by the air and water velocities $V_{1}, V_{0}$ and the angle $\alpha$ between the vectors $\boldsymbol{V}_{1}$ and $\boldsymbol{V}_{0}$. It means that the straight lines $\theta=\beta, \theta=\beta+\pi$ and $\theta=\beta \pm \frac{1}{2} \pi$, characterizing all (by the growth rate) wave length diagrams are obtained by measurements and simplest calculations.

The most important azimuth $\beta( \pm \pi)$ property is that the wave length at this direction equals zero. This follows from the formula (19) and also from the wavelength diagram (Fig. 2). The zero wavelength direction $\theta=\beta$ is easily seen on the water waves air photograph at strong wind (Fig. 1).

The lines $\theta=\beta, \theta=\beta+\pi$ and $\theta=\beta \pm \frac{1}{2} \pi$ are frozen-in the wave plane. They are called the skeleton of the wave length diagram.

\subsection{The wave lengths similarity theorem}

It was mentioned above that at $K=1$ the wind waves are neutral. In this case it follows from the formula (19) that the wind wavelengths at any value of parameter $K$ are similar to the lengths $\lambda_{1}=L_{1}|\sin (\theta-\beta)|$ of the neutral waves for any fixed direction $\theta$. The stated theorem essentially takes into account the fact that the angle $\beta$ does not depend of the $K$ parameter.

The circle $\lambda_{1}=L_{1}|\sin (\theta-\beta)|$ does not depend of the growth rate. That is why it also enters the skeleton of the wind wavelengths diagram.
Note also that the wind wave lengths have all the properties of circle chords. In particular the sum of wave lengths squared in two perpendicular directions equals to the squared maximal waves length:

$\lambda_{K}^{2}(\theta)+\lambda_{K}^{2}\left(\theta \pm \frac{\pi}{2}\right)=L_{K}^{2}$.

\section{Wind waves velocities azimuth hodograph}

From formula $\dot{\lambda}_{K}=\Sigma_{k} \sin \left(\theta+\theta_{K}\right)$ (see Eq. 21) one can see, that the wind waves velocities diagram is also a circle. We are going to call the wind waves velocities diagram azimuth hodograph of the wind waves.

\subsection{Motionless wind waves}

The azimuth hodograph circle of the wind waves touches the straight line $\theta=-\theta_{K}, \theta=-\theta_{K}+\pi$. Therefore at the direction $\theta=-\theta_{K}$ the wind waves velocity equals zero.

In the orthogonal direction $\theta=(\pi / 2) \operatorname{sgn}(K)-\theta_{K}$ (see Eq. 21) the zero velocity troughs are located. We will call the waves with zero velocity as motionless waves. In the motionless wave's troughs foam, driftweed and other minor material from the water surface is accumulated. Thereby the motionless waves are marked on the water surface as motionless light stripes called "the Langmuir stripes". The motionless waves are seen on the water surface air photographs at strong wind (Fig. 1). We do not consider in this paper the Langmuir circulations as it was made in the paper (Craik and Leibovich, 1976), though it should be noted, that our approach is more preferable, since we use both the water current and the wind currant and not just the surface stresses as in the paper (Craik and Leibovich, 1976).

If put aside all the damped neutral and non damped wind waves and leave only the motionless waves, the water surface will become crimped in the direction orthogonal to the Langmuir stripes. The amplitude of such a goffer diminishes at damped waves and increases up to total destruction at non damped waves. The goffer of the neutral wind waves is frozen-in the other waves until the wind blows.

As a result of the wind waves dynamic interaction the motionless Langmuir stripes are described here for the first time.

The center of the wind waves velocities hodograph is situated of the strait line $\theta=(\pi / 2) \operatorname{sgn}(K)-\theta_{K}$ at the distance $\frac{1}{2} \Sigma_{K}$ from the point of contact of the hodograph with the line $\theta=-\theta_{K}, \theta=-\theta_{K}+\pi$ (Fig. 3).

The axis $\theta=(\pi / 2) \operatorname{sgn}(K)-\theta_{K}$ of the wind waves velocities hodograph rotates at the parameter $K$ change (see the formulas (21), defining the trigonometric functions of the angle $\left.\theta_{K}\right)$. That is why there is no wave velocity similarity theorem.

Figure 3 describes one of the possible wind waves velocities azimuth hodographs with the center at the point $C_{K}$. The vector $\boldsymbol{\Sigma}_{K}$ in Fig. 3 equals the maximal wind waves 
velocity for the given parameter $K$ value. The vector $\boldsymbol{O A}$ in Fig. 3 equals the wind waves velocities at the direction $\theta$ (for the given parameter $K$ value). The vector $\boldsymbol{V}_{1}$ equals the wind velocity. All the azimuths are counted out from the vector $\boldsymbol{V}_{1}$ on the $z$-plain. The velocity hodograph diameter directed along the maximal wind waves velocity $\Sigma_{K}$ is called the main diameter of the hodograph.

\section{$5.2 \sigma$-hodograph}

Let us find the wind waves velocity along the direction $\theta=\beta$. Let in the formula (18) $\theta=\beta$ and take into account formulas (16). Then

$\sigma=\frac{\sqrt{A} \sin (\beta+\gamma)}{1+\mathrm{d}}=\frac{V_{1} V_{0} \sin \alpha}{\sqrt{V_{1}^{2}-2 V_{1} V_{0} \cos \alpha+V_{0}^{2}}}$.

Here velocity $\sigma \equiv \lambda_{K}(\beta)$ is the velocity of the zero length waves that is the velocity of the wave ripples in the direction $\theta=\beta$.

$\sigma$-hodograph is the circle which main diameter equals to $\sigma$ and is directed along the azimuth $\theta=\beta$ at $0 \leq \alpha \leq \pi$ and along the azimuth $\theta=\beta+\pi$ at $(-\pi)<\alpha<0$. The $\sigma$-hodograph circle takes the form:

$\dot{\lambda}_{\sigma}=\sigma \cos (\theta-\beta), \quad(0 \leq \alpha \leq \pi)$.

Let us find the minimal value of the velocity $\Sigma_{K}$ with respect to parameter $K$ using the formulas (21) and (16). It turns out as a result that the minimum is obtained at

$K=K_{\sigma}=-\frac{C}{B}$,

and

$$
\min _{K}\left|\Sigma_{K}\right|=\max _{\theta} \min _{K}\left|\dot{\lambda}_{K}\right|=|\sigma| .
$$

This means that the minimal wind waves velocity with respect to $K$ equals $|\sigma|$.

But the main property of the wind waves velocity of zero length is that the velocity $\sigma$ does not depend of the growth rate ${ }^{1}$. That is why since the value $K_{\sigma}$ corresponding to the main diameter of the $\sigma$-hodograph (see Eq. 24) belongs to a set of values of the parameter $K$, the main diameter $\sigma$ of the $\sigma$-hodograph is a chord of any velocity hodograph of the wind waves. Now it is possible to formulate the main theorem of geometric dynamics of the non gravitational wind waves:

The locus of all the centers of the main diameters of the wind waves velocity hodographs is a strait line passing perpendicular to the vector $\sigma$ via its middle.

\footnotetext{
${ }^{1}$ In accordance with the formula (22) the parameter $\sigma$ is calculated after when the velocities of the air and water flows $\boldsymbol{V}_{1}$ and $\boldsymbol{V}_{0}$ and the angle between them have been obtained.
}

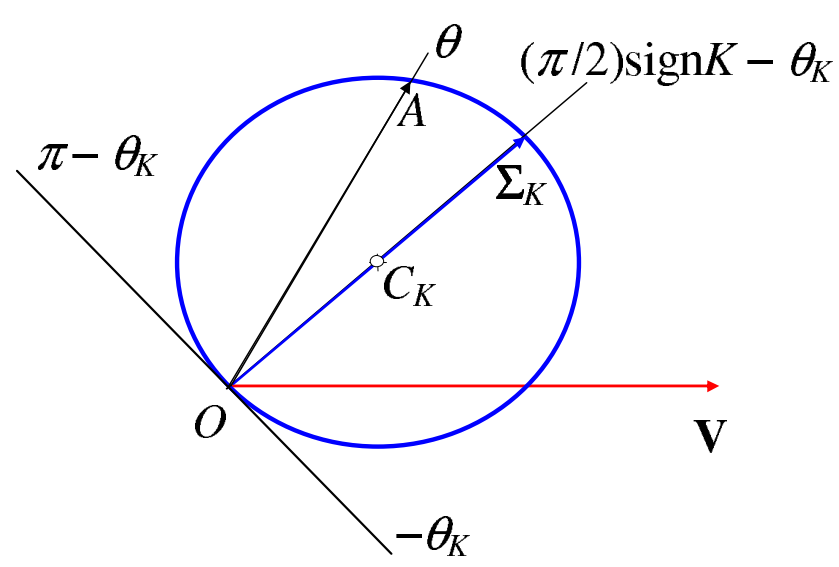

Fig. 3. Wind waves velocities azimuth hodograph.

The locus of all the main diameters ends of the wind waves velocity hodographs is a strait line passing perpendicular to the vector $\sigma$ via their ends.

Consequence.

Since the vector $\sigma$ is oriented along or against the ray $\theta=\beta$, perpendicular to the vector $\sigma$ centers line and the main diameters ends line are parallel to the axis $\theta=\beta \pm \pi / 2$ of the wavelengths diagram.

Therefore, the wavelengths diagram, the main $\sigma$ hodographs diameter and the two lines: the line of the centers of the main velocity hodographs diameters and the line of the ends of the same main diameters enter into the general skeleton of the wind waves dynamics.

The physical meaning of the theorem and its consequence is in the fact that the main $\sigma$-hodograph diameter can be measured by the value and direction ${ }^{2}$.

\section{Absolutely motionless waves, freak waves}

Motionless wind waves in this approach appear in a natural way since the sine in the formula

$\dot{\lambda}_{K}=\Sigma_{K} \sin \left(\theta+\theta_{K}\right)$

always reduces to zero at the direction $\theta=-\theta_{K}$ (see Eq. 21). There appears a question whether wind waves with zero velocity exist at any azimuth value $\theta$. To answer this question let us see at what values of the $K$ parameter reduces to zero the coefficient $\Sigma_{K}(K)$ in the formula (26). Applying once again to the formulas (21) we can see that there are two such values. Their expressions after some simplifications take the form:

$K=K_{0}=\frac{2 \delta\left(V-V_{0}\right)}{\delta V+V_{0}} \quad(\alpha=0)$,

\footnotetext{
${ }^{2}$ The vector $\sigma$ direction is defined by the sign of the function $\sin \alpha$.
} 
$K_{\pi}=\frac{2 \delta\left(V+V_{0}\right)}{\delta V-V_{0}} \quad(\alpha= \pm \pi)$

At these growth rate values the wind waves represent a dynamic barkhan-like water surface deformation. The height of the water barkhans diminishes if the values (27) are less than unity and increase up to overturning at $K_{0}>1$ or $K_{\pi}>1$. If $K_{0}=1$ or $K_{\pi}=1$, the wind born water barkhan are neutral.

The barkhan like waves are encountered in the vicinity of south-east Africa and more rearly along the Gulf Stream flow. In the case when the barkhan like wave's amplitude increases quickly $(K>1)$, the absolutely motionless wave is called freak wave, rogue wave, rabid-dog wave and so on. The waves like this sometimes sink ships. In the south-east Africa region the rogue waves appear on the Agulhas Current when the wind is directly oriented along the water current $(\alpha=0)$. This Agulhas Current and the Agulhas Retroflection can give rise to immense rogue waves that can threaten supertankers. For that reason, mariners who successfully navigated the Cape of Good Hope frequently breathed a sigh of relief. A very well known is the New Year's Day wave, that struck the stationary Draupner oil platform in the North Sea on 1 January 1995. During this event, minor damage was inflicted on the platform, confirming that the reading was valid. From that time it was concidered that once thought to be only legendary, they are now known to be anatural phenomeon, not rare, but rarely encountered. On the Gulf Stream in the region of the Bermuda Islands, the rogue waves appear and gain height of 18 meters. In 1912 in the Bermuda Islands region a three masts sailing vessel was knocked out by a rogue waves of $30 \mathrm{~m}$ high. The next wave sank the vessel.

\section{Wind waves express-forecast}

\section{$7.1 \quad(-2 \delta)$-hodograph}

Let us assume in the formula (20) $K=-2 \delta$. Then obtain the velocity hodograph of the wind waves

$\boldsymbol{v}_{(-2 \mathrm{~d})}=V \cos \theta$

The main hodograph diameter (28) is oriented along the wind vector $\boldsymbol{V}$. The radius of the velocity hodograph circle (28) equals half of the wind velocity. Since $(-2 \delta)<1$, the hodograph (28) (the wind velocity hodograph) belongs to the damped weaves set.

Despite the simplicity of the hodograph (28), it illustrates the main property of the considered waves: the wind waves propagate at many possible directions (in the considered case it is the direction $(-\pi \leq \theta \leq \pi))$. The usual plane waves would propagate only in one direction $(\theta=0)$.

\subsection{2-hodograph}

Assume in the formula (20) $K=2$. Then obtain the velocity hodograph of wind waves

$\boldsymbol{v}_{2}=V_{0} \cos (\theta-\alpha)$.
The main hodograph diameter (29) is oriented along the velocity vector of the underwater current $\boldsymbol{V}_{0}$. The radius of the velocity hodograph circle (29) equals $\frac{1}{2} V_{0}$. Since $2>1$, the hodograph (29) (the underwater current velocity hodograph) belongs to the set of non damped waves.

Both hodographs (28) and (29) can be defined by means of measurements and simplest calculations of the angle $\beta$. Indeed, let us choose on the $z$ plane an arbitrary pole $O$ and draw two vectors from it: vector $\boldsymbol{V}$ of the air velocity and vector $\boldsymbol{V}_{0}$ of the underwater current velocity. Consider the triangle $O \boldsymbol{V} \boldsymbol{V}_{0}$. This triangle can always be plotted since its two sides $\left(V\right.$ and $\left.V_{0}\right)$ and the angle $\alpha$ between them can be measured.

Remind now that the locus of the main diameters of the wind waves velocities is a straight line. Since the vectors $\boldsymbol{V}$ and $\boldsymbol{V}_{0}$ are the main diameters of some hodographs the whole line of the ends of the wind waves hodographs main diameters passes by the ends of the vectors $\boldsymbol{V}$ and $\boldsymbol{V}_{0}$ that is, the line of the wind waves hodographs main diameters ends contains the triangle $O \boldsymbol{V} \boldsymbol{V}_{0}$ base opposite to the $O$ apex.

Now it is clear that the locus of the hodographs centers of the wind waves is the triangle's $O \boldsymbol{V} \boldsymbol{V}_{0}$ centerline parallel to the base $V V_{0}$.

Therefore, one can conlude that the locuses of the centers of the wind waves velocities hodographs and the ends the same diameters are easily determined by means of wind and underwater current velocities measurements.

It is easily understood that the main diameter $\sigma$ of the $\sigma$-hodograph is an oriented triangle $O \boldsymbol{V} \boldsymbol{V}_{0}$ altitude passed from the apex $O$.

\subsection{The main hodographs diameters orientation sectors}

The wind waves dynamics has one peculiarity. As we have just seen, the main diameter $\boldsymbol{V}$ of the hodograph (28) belongs to the region of non damped waves. The main diameter $\boldsymbol{V}_{0}$ of the hodograph (29) belongs to the region of damped waves. What is the hodograph that is the boundary dividing the damped and the non damped waves? At first sight the answer is simple: the boundary is the hodograph $\dot{\lambda}_{1}=\Sigma_{1} \sin \left(\theta+\theta_{1}\right)$ (see Eq. 21) of neutral waves $(K=1)$. Indeed at $K<1$ the wind waves damp and at $K>1$ do not damp right up till destruction a cause of the wave amplitude growth.

So, if we move along the centers of the wind waves hodographs from the azimuth $\theta=\beta-\pi / 2$ to azimuth $\theta=\beta+\pi / 2$, the situation changes. Point is, that the hodograph $K= \pm \infty$, that is, (by the Eq. 18), the hodograph

$\boldsymbol{v}_{\infty}=\frac{\sqrt{A}}{1+\delta} \sin (\theta+\gamma)$

of the wind waves velocities of zero lengths (see Eq. 19) enters the wind waves dynamics. That is why the velocities hodograph (Eq. 30) is located at the certain point $C$ of the velocities hodograph centers line of the wind waves. 


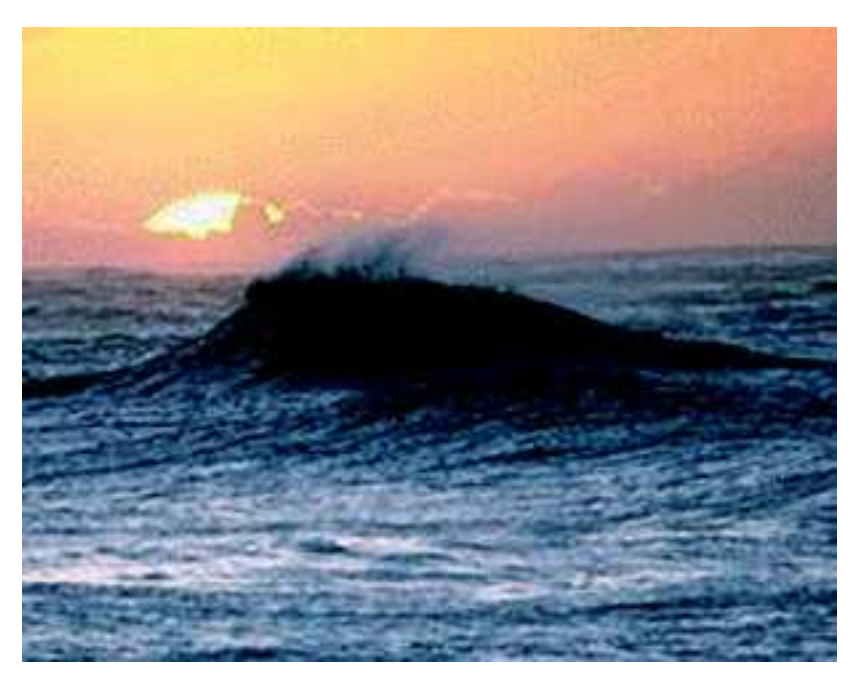

Fig. 4. A freak wave.

Let us mark the center $C$ of the hodograph (Eq. 30) $(K=\infty)$ on the centers line of wind waves velocities hodographs.

If we move along the indicated line of the centers from the point $C(K=\infty)$ to the point $K=1$ we will pass the centers of the velocities hodographs of the non damped waves $(1<K<\infty)$. At $K=1$ we get to the center of the velocities hodograph of neutral waves. At the further decrease of the parameter $K$, at $0<K<1$ we move along the hodograph centers of damped waves. At $K=+0$ we obtain an internal resonance of the growth rate of the waves with the turbulent resistance frequency $c_{i}=1$ or in dimensional form $c_{i}^{\prime}=\kappa$. This will take place on the damped waves.

Note, that at $K=2>1$ the hodograph (29) of the water current velocity enters the velocities hodographs set of non damped waves. The main hodograph (29) diameter is located on the ray $\theta=\alpha$. That is why at $0 \leq \alpha \leq \pi$ the velocities hodographs centers of the wind waves at $K \rightarrow+0$ go upward along the ray ${ }^{3} \theta=\beta+\pi / 2$.

Let us pass now from the point $C(K=-\infty)$ to the point $K=-0$. We will again pass along the hodographs centers of the wind waves velocities but this time along the velocities hodographs centers we go downward along the ray $\theta=\beta-\pi / 2$.

Thus the orientation sectors of the main diameters hodographs of the damped waves at $+0<K<1$ and $-\infty<K<-0$ are divided by the orientation sector of the main hodographs diameters of the damped waves velocities $(1<K<\infty)$. In this sense the damped waves at $+0<K<1$ and at $-\infty<K<-0$ differ from each other and the waves at $+0<K<1$ we will call weakly damped in contrast to strongly damped waves at $-\infty<K<-0$ (see Fig. 5).

\footnotetext{
${ }^{3}$ Motion downward along the ray $\theta=\beta-\pi / 2$ in this case is impossible since at $0 \leq \alpha \leq \pi$ the azimuth $\theta=\beta-\pi / 2<0$
}

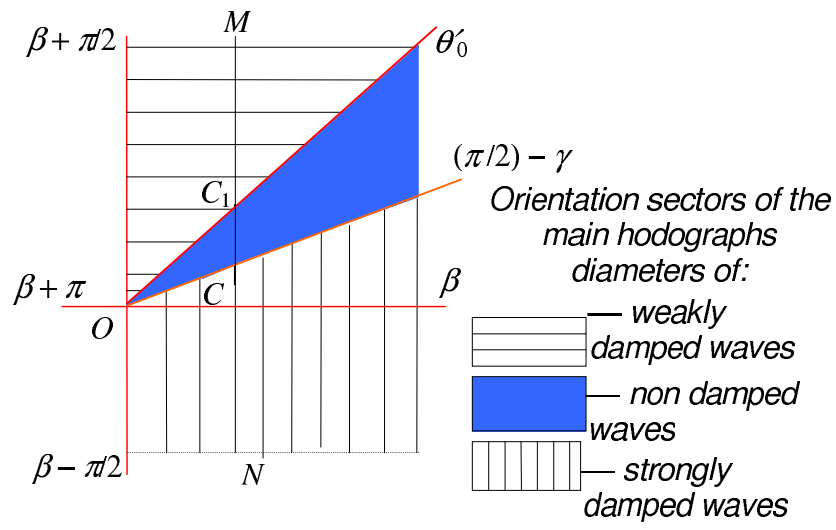

Fig. 5. Distribution of the sectors of the strongly- and weaklydamped waves

In the point of view of the Fig. 5 the destroying nondamped waves are weaker than the damped waves, since the orientation sector of the velocities hodographs of non damped waves occupy smaller part of the wave surface.

Let us take an arbitrary pole $O$ on the $z$-plane. Draw via the pole $O$ a counting out vector $\boldsymbol{V}_{1}$ of the azimuths $\theta$. Than via pole $O$ draw a skeleton $\theta=\beta, \theta=\beta+\pi$ and $\theta=\beta \pm \pi / 2$ of the waves lengths diagram (Fig. 6).

Let us draw a vector $\sigma$ the main diameter of the $\sigma$-hodograph from the pole $O$ along the azimuth $\theta=\beta$ $(0 \leq \alpha \leq \pi)$. Along the azimuth $\theta=\alpha$ from the pole $O$ draw a vector $V_{0}$ of the velocity of the underwater current. Via the ends of the vectors $\boldsymbol{V}$ and $\boldsymbol{V}_{0}$ draw a straight $P Q-$ line of the ends of the main hodographs diameters of the wind waves velocities (Fig. 6).

Draw a line $M N$ via the triangle $O \boldsymbol{V} \boldsymbol{V}_{0}$ centerline of the centers of the main hodographs diameters of the wind waves velocities.

Let us fix the parameter $K$, make for its value a waves lengths diagram and draw a tangent $\theta=-\theta_{K}, \theta=-\theta_{K}+\pi$ to the velocities hodograph of the wind waves with the growth rate $c_{i}=K-1$.

Draw a ray $\theta=\theta_{K}^{\prime}=(\pi / 2) \operatorname{sgn}(K)-\theta_{K}$ via the pole $O$ up to the centers lines and the ends of he main wind waves velocities diameters intersection.

As a result make a main diameter of the velocities hodograph of the wind waves with the given growth rate. The hodograph center is in the intersection point of the line $M N$ with the ray $\theta=\theta_{K}^{\prime}$. The main hodograph diameter end of the velocities is in the intersection point of the line $M N$ with the same ray $\theta=\theta_{K}^{\prime}$ (Fig. 6).

Knowing the ray $\theta=\theta_{K}^{\prime}$ let us define the maximum wind waves velocity $\Sigma_{K}$ of the given growth rate and their length $\ell_{K}=O D$ (Fig. 6):

$$
\ell_{K}=L_{K}\left|\sin \left(\theta_{K}^{\prime}-\beta\right)\right|
$$




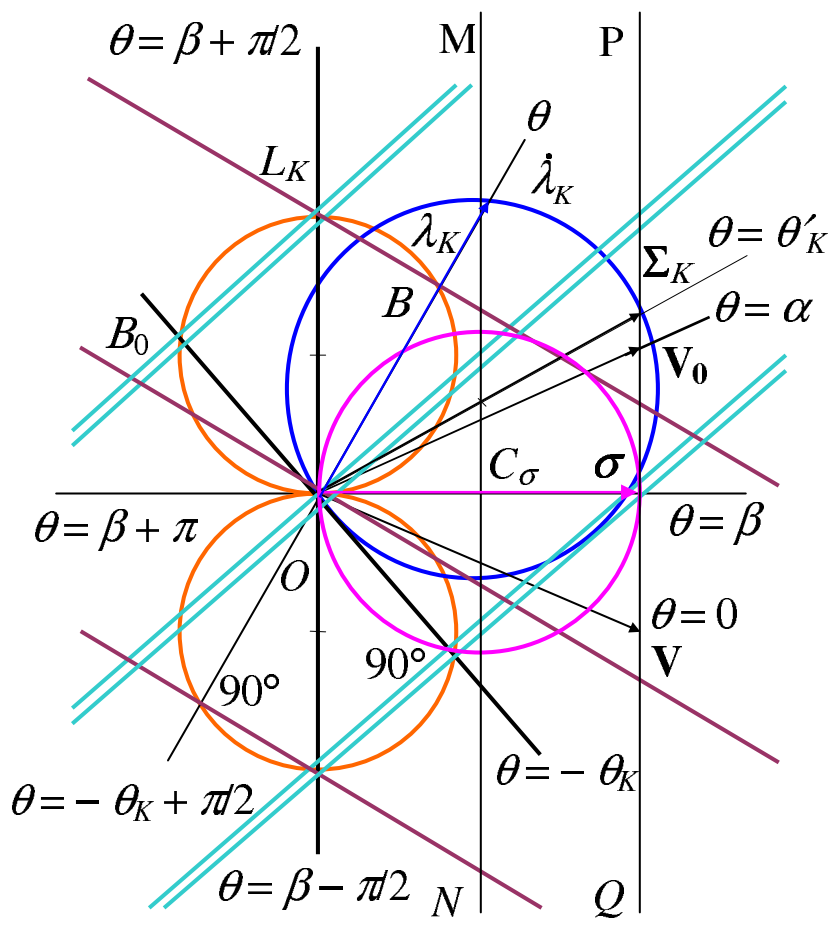

Fig. 6. The wind waves express forecast.

In the intersection point of the strait line $\theta=\beta \pm \pi / 2$ with the velocities find the velocity $\sigma_{K}$ of the waves of maximal length

$\sigma_{K}=\Sigma_{K} \cos \left(\theta_{K}+\beta \pm \pi / 2\right)$

Having fixed some direction $\theta$ (Fig. 6), find the velocity $\boldsymbol{O} \boldsymbol{A}$ and the wave length $O B$ in this direction.

The non gravitational wind waves have one more interesting property: the waves of the equal velocity value can have different wave lengths. The waves of equal wave length can have different velocities.

For example, in the direction $\theta=\beta$, the wave velocity equals $\sigma$, and the wave length equals zero. The waves symmetric with respect to the axis $\theta=\theta_{K}^{\prime}$ of the hodograph have the same velocity value $O E$, but not the same wave length $O F$.

The neutral waves $(K=1)$ of the equal velocity and different wave length represent choppy sea. The problem of choppy sea has not been yet described analytically. Here this problem is solved with the use of a couple of circles.

The double lines on the Fig. 6 mark the Langmuir stripes that are evidently parallel to the main hodograph diameter $\Sigma_{K}$ of the wind waves velocities at the given value of $K$. The lines $L_{K} B, B_{0} O$ and others (Fig. 6) represent the troughs lines of the wind waves propagating with velocity $\dot{\lambda}_{K}$ along the azimuth $\theta$.

\section{Conclusions}

A common practice in both theoretical and practical hydrodynamics is to consider sea waves as gravitational. Results presented in this work at least qualitatively coincide with both visual observations and with air-photography observations, therefore assure us that the main sea and river waves have not the gravitational but turbulent nature: the initial relations (14) do not contain the acceleration $g$. These relations have a profound hydrodynamic nature - cluster and microheterogeneous liquid structure and as the consequence their turbulent resistance - the main wind waves stimulator, since in its absence $\left(\kappa=\kappa_{1}=0\right)$ it is impossible to formulate the initial non disturbed adjacency current (2). It is quite natural, that though the approximately obtained dispersion relation (14), proves to be so informative. These relations not only explain all the main properties of the considered waves (variety, crestedness, propagation direction, velocity, wave length, choppy sea etc.), but also reveal new - motionless - waves that are marked on the water surface by driftweed, foam as light Langmuir stripes, and thus are observed on the water surface in windy weather as real motionless waves. The value of turbulent resiatance $\kappa$ for air and water have to be measured experimentally. Recently, we obtained the value for $\kappa$ in case of turbulent flow of water through a pipe (to be published). The value is $\kappa=0.0231 / \mathrm{s}$. Using this value for both water and air turbulent resistance, and reasonable estimation for wind velocity $(10 \mathrm{~m} / \mathrm{s})$, water velocity $(1 \mathrm{~m} / \mathrm{s})$, and $\alpha=30^{\circ}$ one obtains from (19) estimation of the wavelength of the Langmuir waves approximately $21 \mathrm{~m}$. More precise values are subject of further experimental work.

Physically the multiple order roots of dispersion relation means more profound penetration of the influence of surface effects on the profound water layers. In this sense the turbulent part $\xi_{1}=\xi_{2}$ of the dispersion relation is more important than the other parts of (14), including gravitational waves. The cause for this is the soundness of the description of the wind properties on the water surface resulting from these simple relations. The apparent three-dimensional wave chaos on the water surface in reality is fully regulated and in order to understand it one needs to know two angles: $\theta=\beta, \theta=\theta_{K}^{\prime}$ and three circles: wave lengths diagrams and $\kappa$-hodograph of their velocities. Express information of the turbulent waves on the water surface is given only by three vectors: the wind velocity $\boldsymbol{V}_{1}$, the water current $\boldsymbol{V}_{0}$ and the vector $\sigma$, direction $\theta=\beta$ ( or $\theta=\beta+\pi)$ of which can be determined by visual observation and its magnitude is equal to the velocity of the wave front-line in this direction. That is why application of the obtained results into practice can be done on-the-fly by taking the wave characteristics (wave lengths, their velocities, propagation directions) on the monitors screens in ship deck-cabins, in dispatching offices of sea ports, drilling plants on shelves, lighthouses, etc. 
Edited by: U. Harlander

Reviewed by: two anonymous referees

\section{References}

Birkhoff, G.: Hydrodynamics, Princeton, New Jersey, Princeton University Press, 1960.

Cherkesov, L. V.: Hydrodynamics of waves, Kiev: "Naukova Dumka", 260 pp., 1980 (in Russian).

Craik, A. D. D. and Leibovich, S.: A rational model for Langmuir circulations, J. Fluid Mech., 73, 4001-426, 1976.

Gill, A. E.: Atmosphere-Ocean Dynamics, Academic Press, London Ltd., 1982.

Kochin, N. E., Kibel, I. A., and Roze, N. V.: Theoretical Hydromechanics, Interscience Publishers, John Wiley \& Sons, 1964.

Kondrat'ev, K. Ya., Nikanorov, A. M., Pantuhin, Ya. V., and Zavolzhensky, M. V.: Ekman drift and other currents in unbounded regions, Doklady Akademii Nauk USSR, 310(5), 1070-1074, 1990.
Lamb, H.: Hydrodynamics, Cambridge, The Univ. Press, 1932.

Monin, A. S.: Hydrodynamics of atmosphere, ocean and Earth interior, St. Petersburg, Hydrometeoizdat, 524 pp., 1999.

Nekrasov, A. I.: Exact theory of steady waves on the heavy fluid surface, Collection of works, V I. Phys. Math. Ed., 358-439, 1961.

Sajjadi, S. G., Thomas, N. H., and Hunt, J. C. R.: Wind-over-Wave Couplings: Perspectives and Prospects, Oxford University Press, USA, 384 pp., 1999.

Stocker, J. J.: Water Waves, Pure and Applied Mathematics, Vol. 9, The Mathematical Theory and Applications, Institute of Mathematical Sciences, New York University, USA, 291-314, 1957.

Struminskii, V. V.: On the theory of Boltzmann kinetic equation, RGD, Vol. 1, VI Int. Symp., N.Y., Akad. Press, 1969.

Titov, L. F.: Wind waves, Hydro. Meteo. Ed., 294 pp., 1969 (in Russian).

Zavolzhensky, M. V. and Rutkevich, P. B.: Large Reynolds Numbers: contour flows, Pr-2117 M., IKI RAS, 60 pp., 2006. 